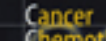

Chemotherapy and

Pharmacology

\title{
Quercetin enhances 5-fluorouracil-induced apoptosis in MSI colorectal cancer cells through p53 modulation
}

\begin{tabular}{|r|l|}
\hline Journal: & Cancer Chemotherapy and Pharmacology \\
\hline Manuscript ID: & CCP-11-0046.R1 \\
\hline Manuscript Type: & Original Article \\
\hline Date Submitted by the \\
Author: & n/a \\
\hline $\begin{array}{r}\text { Complete List of Authors: } \\
\text { Keywords: }\end{array}$ & $\begin{array}{l}\text { Xavier, Cristina; University of Minho, Department of Biology } \\
\text { Rohde, Mikkel; Institute of Cancer Biology, Danish Cancer Society, } \\
\text { Apoptosis Department } \\
\text { Pereira-Wilson, Cristina; University of Minho, Department of Biology }\end{array}$ \\
\hline &
\end{tabular}

\section{SCHOLARONE \\ Manuscripts}


Quercetin enhances 5-fluorouracil-induced apoptosis in MSI colorectal cancer cells through p53 modulation

Cristina P.R. Xavier ${ }^{1}$, Cristovao F. Lima ${ }^{2}$, Mikkel Rohde ${ }^{3}$ and Cristina Pereira-Wilson ${ }^{1, *}$

${ }^{1}$ CBMA - Centre of Molecular and Environmental Biology/Department of Biology, School of Sciences, University of Minho, 4710-057 Braga, Portugal

${ }^{2}$ CITAB - Centre for the Research and Technology of Agro-Environmental and Biological Sciences/Department of Biology, School of Sciences, University of Minho, 4710-057 Braga, Portugal

${ }^{3}$ Apoptosis Department, Institute of Cancer Biology, Danish Cancer Society, Strandboulevarden 49, DK-2100 Copenhagen, Denmark

* Corresponding author: Tel.: +351 253604318; fax: +351 253678980; e-mail address: cpereira.bio.uminho@gmail.com (C. Pereira-Wilson). 


\section{Abstract}

2 Purpose: Colorectal tumors (CRC) with microsatellite instability (MSI) show resistance to 3 chemotherapy with 5-fluorouracil (5-FU), the most widely used pharmacological drug for

4 CRC treatment. The aims of this study were to test the ability of quercetin (Q) and luteolin (L)

5 to increase sensitivity of MSI CRC cells to 5-FU and characterize the dependence of the 6 effects on cells' p53 status.

7 Methods: Two MSI human CRC derived cell lines were used, CO115 wild-type (wt) for p53 8 and HCT15 that harbors a p53 mutation. Apoptosis induction in these cells by 5-FU, Q and L 9 alone and in combinations were evaluated by TUNEL and western. The dependence on p53 of 10 the effects was confirmed by small interference RNA (siRNA) in CO115 cells and in MSI 11 HCT116 wt and p53 knockout cells.

12 Results: CO115 p53-wt cells are more sensitive to 5-FU than the p53 mutated HCT15. The combination treatment of 5-FU with L and Q increased apoptosis with a significant effect for

$14 \mathrm{Q}$ in CO115. Both flavonoids increased p53 expression in both cell lines, an effect particularly remarkable for Q. The significant apoptotic enhancement in CO115 incubated with Q plus 5-FU involved the activation of the apoptotic mitochondrial pathway. Importantly, knockdown of p53 by siRNA in CO115 cells and p53 knockout in HCT116 cells totally abrogated apoptosis induction, demonstrating the dependence of the effect on p53 modulation by Q.

Conclusion: This study suggests the potential applicability of these phytochemicals for enhancement 5-FU efficiency in MSI CRC therapy, especially Q in p53 wt tumors.

25 Keywords: Apoptosis; Colorectal carcinoma; 5-fluorouracil; p53; Quercetin 


\section{Introduction}

Chemotherapy with 5-fluorouracil (5-FU) is the basis for colorectal carcinoma (CRC) treatment, one important cause of cancer related death in western societies [1]. However, significant resistance to this drug has been reported [2-4]. To overcome resistance, drugs such as irinotecan and oxaliplatin are used in combination with 5-FU and have provided increased efficacy although not in all patients $[3,5,6]$. Particularly, tumors with microsatellite instability (MSI) do not generally respond satisfactorily to 5-FU [7-10]. MSI tumors, which occur in approximately $15 \%$ of sporadic CRC cases and in $90 \%$ of hereditary non-polyposis colorectal cancer (HNPCC), have mutations in the mismatch repair (MMR) genes, resulting in an inability to correct DNA replication errors and in the accumulation of mutations $[8,9,11]$. In vitro studies have also shown that DNA MMR deficiency may be responsible for tumor resistance to $5-\mathrm{FU}[7,8,10]$.

Previous studies have shown that, independently of MSI status, mutations in the gene P53 contribute to 5-FU resistance in CRC and have profound effects on drug responses $[12,13]$ with reduced induction of apoptosis and inhibition of cell cycle $[14,15]$. The prognosis in patients presenting MSI tumors with p53 mutations have been shown to be poorer compared to those having MSI tumors with p53 wild-type [16].

The induction of apoptosis by 5-FU may occur through p53 activation and both the intrinsic and extrinsic pathways with activation of caspases [17]. In the intrinsic pathway, Bcl-2 family proteins modulate mitochondrial membrane permeabilization, which leads to the release of cytochrome $\mathrm{c}$ and activation of caspase- 9 that in turn activates the effector caspase3. Activation of death receptors on the cell membrane (extrinsic pathway), which subsequently activates caspase-8 and caspase-3, may also be induced by 5-FU [17].

Dietary phytochemicals have been shown to induce apoptosis through modulation of different pathways contributing to decrease tumor malignance and chemoresistance [18-20]. 
51 In addition to effects on mitochondrial and death receptors pathways, some proapoptoitc

52 effects of natural compounds have also been attributed to c-Jun N-terminal kinase (JNK) and p38 stress-activated protein kinases [19,21-23].

In a previous study, we showed that two structurally related flavonoids quercetin $(Q ;$ a

flavonol) and luteolin (L; a flavone), commonly found in fruits and vegetables, have antiproliferative effects in HCT15 (mutant KRAS) and CO115 (mutant BRAF) human CRC cells through regulation of KRAS and both the MAPK/ERK and the PI3K pathways [24]. The anticarcinogenic effects of these flavonoids suggest the suitability of diet rich in Q and/or L for CRC patients undergoing treatment with 5-FU. In the present study, we tested the possible therapeutic efficacy of Q and L in combination with the pharmaceutical drug 5-FU in a MSI p53 wild-type (wt) and a p53 mutant CRC cell lines, CO115 and HCT15, respectively $[25,26]$. In addition, the mechanism of $\mathrm{Q}$ and $\mathrm{L}$ in inducing apoptosis in these MSI CRC cell lines was investigated and the dependence on p53 confirmed by siRNA. Our data shows the potential applicability of these flavonoids for use in combination with 5-FU to induce apoptosis in CRC, particularly for Q in a p53 wt background.

\section{Material and methods}

\section{Reagents and antibodies}

Quercetin (Q), z-VAD-fmk (zVAD), staurosporine (STS), 5-fluorouracil (5-FU) and 3-(4,5-Dimethylthiazol-2-yl)-2,5-diphenyltetrazolium bromide (MTT) were purchased from Sigma-Aldrich (St. Louis, MO, USA). Luteolin (L) was purchased from Extrasynthese (Genay, France). Stock solutions of test compounds were made in dimethyl sulfoxide (DMSO) and aliquots were kept at $-20^{\circ} \mathrm{C}$. All other reagents and chemicals used were of analytical grade. 
Primary antibodies were purchased to the following sources: anti-cleaved caspase-9 and anti-phospho-p38 MAPK (Thr180/Tyr182) to Cell Signaling (Danvers, MA, USA); anticaspase-3 to Calbiochem (San Diego, CA); anti-Bcl-2, anti-Bax, anti-PARP-1, anti-phosphoJNK, anti-JNK, anti-p38 total and anti-p53 to Santa Cruz Biotechnology, Inc. (Santa Cruz, CA, USA) and anti- $\beta$-actin to Sigma-Aldrich. Secondary antibodies HRP donkey anti-rabbit and sheep anti-mouse were purchased to GE Healthcare (Bucks, UK).

\section{Cell lines}

HCT15 and CO115 human colon carcinoma-derived cell lines were kindly provided by Dr. Raquel Seruca (IPATIMUP, University of Porto, Portugal. The two isogenic HCT116 colon carcinoma, p53-wild type (p53-wt) and p53 complete knockout for p53 (p53-null) cells were kindly provided by Vogelstein [27]. The cell lines were maintained at $37^{\circ} \mathrm{C}$ in a humidified 5\% $\mathrm{CO}_{2}$ atmosphere in RPMI-1640 medium (Sigma-Aldrich) supplemented with 10mM HEPES, 0.1mM pyruvate, $1 \%$ antibiotic/antimycotic solution (Sigma-Aldrich) and 6\% heat-inactivated fetal bovine serum (FBS; EU standard, Lonza, Verviers, Belgium).

\section{Cell viability/proliferation assay}

To investigate the effects of 5-FU on cell viability/proliferation in HCT15 and CO115 cells, as well as, the effect of Q and 5-FU in the two isogenic HCT116 cell lines, the MTT reduction assay were used as described previously [24]. Cells were treated with different concentrations of 5-FU and Q for 46h and then two more hours in the presence of MTT (final concentration $0.5 \mathrm{mg} / \mathrm{ml}$ ). Hydrogen chloride $0.04 \mathrm{M}$ in isopropanol was then used to dissolve the formazan crystals. The number of viable cells in each well was estimated by the cell capacity to reduce MTT, using a spectrophotometer. The results were expressed as percentage relative to the control (cells without any test compound), and MTT reduction at the beginning 
101 of incubation ( $\mathrm{t}=0 \mathrm{~h})$ was subtracted from all experimental conditions, including the control.

102 Since the effects of the compounds were studied in $48 \mathrm{~h}$ incubations and cells grow 103 significantly during this period, this treatment of the results allows to distinguish between 104 significant cell death (negative values) and inhibition of proliferation (values between 0 and $105100 \%)$. The IC50 corresponds to the concentration that inhibits cell viability/proliferation by $10650 \%$. Results are presented as mean \pm SEM of at least three independent experiments.

107

108

109

110

TUNEL assay

TUNEL (TdT mediated dUTP Nick End Labelling) assay was performed to estimate the percentage of apoptotic cells treated for $48 \mathrm{~h}$ with different concentrations of 5-FU alone and in combination with Q and L. In HCT15 and CO115 cell lines, the concentrations of Q and L used induce significant inhibition of cell proliferation and cell viability without substantial severe and acute cell death, as determined by BrdU assay and MTT test in a previous study using the same cells and conditions [24]. Both cell lines were also treated with Q and L in combination with $20 \mu \mathrm{M} z-\mathrm{VAD}-\mathrm{fmk}(\mathrm{zVAD})$, a general caspase inhibitor, for 48h, to assess the involvement of caspases activation in the apoptotic process induced by the test compounds. Staurosporine (STS) $0.25 \mu \mathrm{M}$, an apoptosis inducer, was also used as a positive control. In HCT116 isogenic cell lines (p53 wt and p53-null) and CO115 cells depleted for p53 by small interference RNA (siRNA), the concentrations of 5-FU and Q used significantly inhibited cell proliferation without substantial severe and acute cell death.

After treatments, cells were collected (both floating and attached cells), fixed with $4 \%$ paraformaldehyde for $15 \mathrm{~min}$ at room temperature and attached onto a polylysine treated slide using a Shandon Cytospin. Cells were then washed in PBS and permeabilized with $0.1 \%$ Triton $\mathrm{X}-100$ in $0.1 \%$ sodium citrate for $2 \mathrm{~min}$ on ice. TUNEL assay was performed using a kit from Roche (Mannheim, Germany), following the manufacturer's instructions. Hoechst 
126 was used for nuclei staining. The percentage of apoptotic cells was calculated from the ratio

127 between TUNEL positive cells and total number of cells, from a count higher than 500 cells

128 per slide under a fluorescent microscope. Results are presented as mean \pm SEM of at least

129 three independent experiments.

131 Western blot analysis

132 Cells were treated with Q, L, 5-FU and STS alone and co-incubated with Q and 5-FU

133 for $48 \mathrm{~h}$ and total cell lysates were prepared to measure the expression of different proteins.

134 The cells were washed with PBS and lysed for $15 \mathrm{~min}$ at $4^{\circ} \mathrm{C}$ with ice cold RIPA buffer $(1 \%$

135 NP-40 in 150mM NaCl, 50mM Tris (pH 7.5), 2mM EDTA), supplemented with 20mM NaF,

$1361 \mathrm{mM}$ phenylmethylsulfonyl fluoride (PMSF), $20 \mathrm{mM} \mathrm{Na} \mathrm{V}_{3} \mathrm{O}_{4}$ and protease inhibitor cocktail

137 (Roche, Mannheim, Germany). Protein concentration was quantified using a Bio-Rad DC

138 protein assay (Bio-Rad Laboratories, Inc., Hercules, CA, USA) and BSA used as a protein

139 standard. To perform western blot analysis, $20 \mu \mathrm{g}$ of protein were resolved by SDS-

140 polyacrylamide gel and then electroblotted onto a Hybond-P polyvinylidene difluoride

141 membrane (GE Healthcare). Membranes were blocked in TPBS (PBS with 0.05\% Tween-20)

142 containing $5 \%(\mathrm{w} / \mathrm{v})$ non-fat dry milk or BSA (bovine serum albumin), washed in TPBS and

143 then incubated with primary antibody. After washing, membranes were incubated with

144 secondary antibody conjugated with IgG horseradish peroxidase and immunoreactive bands

145 were detected using the Immobilon solutions (Millipore, Billerica, MA, USA) under a

146 chemiluminescence detection system, the Chemi Doc XRS (Bio-Rad Laboratories, Inc.).

147 Band area intensity was quantified using the Quantity One software from Bio-Rad. $\beta$-actin 148 was used as loading control.

149

$150 \quad$ p53 knokdown in CO115 cells 
CO115 human colon cancer cells were transiently transfected with Oligofectamine

152 (Invitrogen, Carlsbad, CA) and 100nM of p53 siRNA in OPTI-MEN (Invitrogen) according

153 to the manufacturer's instructions. The p53 siRNA target sequence was

154 guggaguauuuggaugaca, which was purchased from Invitrogen. Control of siRNA experiments

155 were included by using a siRNA against GFP. Confirmation of p53 knockdown was done by

156 western blot analysis. Twenty four hours after transfection, cells were incubated with 5-FU or

157 Q alone and with both in combination, for $48 \mathrm{~h}$, and apoptosis measured by the TUNEL assay.

158

159 Statistical analysis

160 Statistical analyses were done using $t$-test, GraphPad Prism 4.0 software (San Diego,

161 CA, USA). $P$-values $\leq 0.05$ were considered statistically significant.

162

163

Results

164

165

Colon cancer cells` sensitivity to 5-FU

166

The effect of 5-FU on cell viability/proliferation and apoptosis in HCT15 and CO115

167 cells were established by the MTT and TUNEL assays, respectively. As shown in Fig. 1a, 5-

168 FU was more effective in decreasing cell viability/proliferation in CO115 than HCT15 after

$16948 \mathrm{~h}$ treatment. The 5-FU concentrations that inhibit cell viability/proliferation by 50\% (IC50)

170 are around $100 \mu \mathrm{M}$ in $\mathrm{HCT} 15$ and $1 \mu \mathrm{M}$ in CO115. The differences in susceptibility of the two

171 cell lines to 5-FU were also observed for apoptosis, with HCT15 being more resistant to

172 apoptosis compared to CO115 (Fig. 1b). IC50 concentrations of 5-FU were selected for the

173 next experiments (100 $\mu \mathrm{M}$ for $\mathrm{HCT} 15$ and $1 \mu \mathrm{M}$ for CO115).

174

175 Combined effect of 5-FU and test compounds on apoptosis 
The induction of apoptosis in both cell lines treated with Q or L, at IC50

177 concentrations, as tested previously [24], or 5-FU (IC50), was monitored by the TUNEL

178 assay in both cell lines. As shown in Fig.2, the flavonoids induced a higher percentage of 179 apoptotic cells in CO115 when compared with HCT15. In HCT15 cells, L or Q in

180 combination with 5-FU demonstrated an additive effect on the induction of apoptosis, i.e., the

181 effects of the combination was similar to the sum of the effects of Q and 5-FU when used

182 alone (Fig. 2a). In CO115 cells, L in combination with 5-FU showed an additive effect in 183 apoptosis induction whereas Q demonstrated to significantly potentiate the induction of 184 apoptotic cell death when combined with 5-FU (Fig. 2b). In all cases, the effects on apoptosis 185 of co-incubations were higher than 5-FU alone or test compound alone.

\section{Effects of Q, L and 5-FU on markers of pathways related with apoptosis}

In order to determine the role of caspase activation on the apoptotic effects of the test compounds, the caspase inhibitor zVAD was used and apoptosis measured by TUNEL assay. Apoptosis induced by the test compounds, Q and L, and STS, the apoptosis inducer, were

191 inhibited by zVAD in CO115 (Fig. 3b) but not in HCT15 (Fig. 3a). In addition, as shown in

192 Fig. 3c, none of the compounds induced cleaved (active) caspase-9 in HCT15 cells, and only

193 STS induced cleavage of caspase-3 (active form) and of PARP (inactive form). On the other

194 hand, in CO115 cleaved caspase-9 and caspase-3 were observed with all compounds as well 195 as cleavage of PARP and/or a remarkable decreased of uncleaved PARP (active form).

To further elucidate the apoptotic effects of the test compounds and 5-FU, the

197 expression of the positive mediators of apoptosis, p53 and Bax, as well as the negative 198 regulator, Bcl-2, were analysed by western blot (Fig. 4a). It was observed that Q, L and 5-FU 199 induced p53 levels in both cell lines. Levels of Bcl-2 were notably decreased by all the 200 compounds in both cell lines. On the other hand, Bax levels were increased by all the 
compounds in HCT15, although only slightly by Q. Bax was not detected in CO115, which is in accordance to a previous report [28].

In addition, the possible involvement of the JNK and p38 pathways on the induction of apoptosis by the test compounds and 5-FU were evaluated. The results show no effect on phospho-JNK levels by Q, L and 5-FU in neither of the cell lines (Fig. 4b). In HCT15 cells no effect on phospho-p38 levels was observed by the flavonoids and 5-FU while in CO115 cells Q and L slightly increased the levels of phospho-p38 (Fig. 4c). STS significantly induced phospho-JNK levels and decreased the expression of phospho-p38 in both cell lines.

\section{Combination of $Q$ and 5-FU: dependence on p53}

Since a significant enhancement of 5-FU-induced apoptosis was observed in CO115 p53-wt cells with Q, an effect concomitant with a robust p53 induction, we further investigated this effect. First, as shown in Fig. 5, combination of Q with 5-FU in CO115 cells also remarkably increased the cleavage of caspase-3, caspase-9 and PARP and decreased Bcl2 expression, but no further induction of p53 was observed. These results indicate that the apoptosis enhancement of Q with 5-FU is at the mitochondrial caspase pathway.

To explore the involvement of p53 in the induction of apoptosis by Q and 5-FU, CO115 cells were depleted for p53 (around 80\%) by siRNA. As shown in Fig. 6a, p53depleted CO115 cells were significantly resistant to apoptosis when incubated with Q or 5FU. In addition, the synergy between Q and 5-FU on the induction of apoptosis was absent in the p53 knockdown cells.

The role of $\mathrm{p} 53$ in the induction of apoptosis by $\mathrm{Q}$ was further confirmed by using two isogenic KRAS activated HCT116 CRC cell lines, one p53-wt and the other with a complete knockout of p53 (p53-null). These cells were incubated with 5-FU or Q alone or with both in combination, at concentrations that induce significant inhibition of cell proliferation without 
226 substantial necrotic death (as observed by the MTT assay; data not shown). HCT116 p53-wt

227 cells were much more susceptible than HCT116 p53-null cells to apoptosis induced by Q and 228 5-FU (Fig. 6b). As in CO115 (KRAS wt), a synergy between Q and 5-FU in the induction of 229 apoptosis was observed in the KRAS mutated HCT116 p53-wt cells.

\section{Discussion}

5-Fluorouracil (5-FU) is the pharmaceutical drug most commonly used in CRC chemotherapy, however, tumor cell resistance to this drug remains a significant concern. Failure to induce apoptosis has been reported to reduce the efficacy of 5-FU, particularly in tumors presenting MSI and/or mutant p53 [7,13,15]. Thus, new compounds are needed to use

236 in combination with 5-FU in order to increase treatment efficacy. In a previous paper, we 237 reported that $\mathrm{Q}$ and $\mathrm{L}$, two structurally related dietary flavonoids, possess potential 238 anticarcinogenic effects in two MSI resistant CRC cell lines, HCT15 and CO115, through 239 inhibition of PI3K/Akt and MAPK/ERK pathways [24]. Inhibitory effects on these two 240 pathways have showed to contribute to an induction of apoptosis and to sensitize to 241 chemotherapeutic drugs [29-33]. Here, we tested these flavonoids in combination with 5-FU 242 and an enhancement of apoptosis was found. HCT15 and CO115 cell lines showed different 243 susceptibilities to 5-FU. As expected, HCT15 cells, harboring a p53 mutation, were more 244 resistant to 5-FU than CO115 cells (wt for p53). Q (in HCT15 cells) and L (in both cell lines) 245 in combination with 5-FU showed to increase apoptosis additively. A significant potentiation 246 of apoptosis induction was detected when treating CO115 p53-wt cells with Q and 5-FU, 247 which indicates a synergy between these two compounds in CO115. The effect on apoptosis 248 of this combination was even more pronounced than that of a 100 times higher concentration 249 of 5-FU when tested alone. 
In CO115 cells, we observed that the caspase inhibitor zVAD totally abrogated

apoptosis induction by $\mathrm{Q}$ and $\mathrm{L}$, which was in agreement with the expression of apoptoticassociated molecular markers, such as cleaved (activated) caspase-9 and caspase-3 as well as a decrease in Bcl-2 expression. These results indicated that $\mathrm{Q}$ and $\mathrm{L}$ induce apoptosis via caspase dependent pathway in CO115 cells with a contribution of the mitochondrial pathway.

Even though CO115 cells do not express Bax, apoptosis induction mediated by mitochondria occurs possibly through interaction with the pro-apoptotic protein Bak. The induction of apoptosis by 5-FU in these cells was low, but also caspase dependent. A remarkable synergy was found for the co-incubation of Q with 5-FU in CO115. In these cells, Q showed a more dramatic induction of p53 compared to $\mathrm{L}$, indicating a possible implication of p53 in the synergy observed between Q and 5-FU in CO115. The co-incubation of Q with 5-FU also remarkably enhanced cleavage of caspase 9, caspase 3 and PARP as well as decreased Bcl-2 levels, compared with each of the compounds alone, suggesting the involvement of the apoptotic mitochondrial pathway in the synergy observed for Q and 5-FU in CO115. This synergy was not observed for HCT15 (p53 mutated) cells which corroborates the dependence of Q on p53 for the observed effect.

In order to elucidate the dependence on p53 of the apoptosis induced by Q and 5-FU, p53 expression was decreased by siRNA in CO115 cells. A phenotype more resistant to apoptosis was observed, and interestingly, the synergy between Q and 5-FU observed in control cells (transfected cells with no p53 silencing) was lost in CO115 cells after p53 270 knockdown. This dependence on p53 was further confirmed using two isogenic MSI (KRAS 271 mutated) HCT116 cell lines. HCT116 p53-null cells showed a smaller apoptotic response to Q 272 and 5-FU as compared to HCT116 p53-wt cells. Furthermore, apoptosis was significantly 273 enhanced when Q was combined with 5-FU only in HCT116 p53-wt cells, with the effect 274 being lost in the HCT116 p53-null cells. These results clearly indicate that Q induces 
275

276

277

278

apoptosis through modulation of $\mathrm{p} 53$, and that this contributes to the synergy found for the combination with 5-FU in p53-wt CRC cells. Moreover, the dependence on p53 in induction of apoptosis by Q seems to be independent of KRAS status, since this effect was observed in CO115 KRAS-wt cell line as well as in the KRAS mutant HCT116 cell line.

The resistance to 5-FU of TP53-deficient CRC cells has previously been reported [15]. In some studies, this chemotherapeutic drug has been combined with natural compounds in different genetic backgrounds, such as triptolide and rosiglitazone in microsatellite stable (MSS) CRC cell lines [34,35] and notoginseng and its ginsenosides in MSI HCT116 p53-wt cells [36], with favourable outcome. Moreover, luteolin was also demonstrated by others [37] to increase the apoptotic effect of the chemotherapeutic drug, cisplatin, in a p53 dependent manner in different cell types including in HCT116 p53-wt cells.

In HCT15 cells zVAD did not inhibit apoptosis induced by any of the test compounds or the reference inducer STS. The lack of caspase-dependent apoptosis was corroborated by the absence of cleaved caspase- 9 and caspase- 3 when these cells were incubated with Q, L or 5-FU. Although all compounds induced p53 expression in HCT15 cells, alteration in the expression of p53 protein is not expected to be of functional significance for apoptosis in this p53 mutated cell line. Also, Bax expression levels increased and Bcl-2 decreased in response to test compounds which, however, did not activate apoptosis through mitochondrial caspase pathway in HCT15 cells. JNK and p38 pathways seem not to be involved in the induction of apoptosis in these cells, contrarily to what was observed with CO115 where the p38 pathway may contribute to the induction of apoptosis. An induction of JNK phosphorylation and a decrease on p38 expression was observed for STS in both cell lines. The activation of JNK by STS has been reported in breast cancer cells [38] but the effect of this compound on these two stress activated kinases in CRC is not well established. 
CRC MSI patients require treatment alternatives that enhance 5-FU responsiveness

300 and would gain from customized treatment modalities based on p53 status. The present study

301 shows the potential applicability of Q and L in the enhancement of the apoptotic effects of 5-

302 FU in MSI CRC cells. Of particular relevance, Q shows the ability to cooperate with 5-FU to

303 potentiate the induction of apoptosis in p53 wt colorectal cancer cells through p53 signaling.

304

305 Acknowledgements

306 CPRX was supported by the Foundation for Science and Technology (FCT), Portugal,

307 through the grant SFRH/BD/27524/2006 and the work was supported by the FCT research

308 grant PTDC/AGR-AAM/70418/2006.

309

310 Conflict of interest

$311 \quad$ None. 


\section{References}

1. Rupnarain C, Dlamini Z, Naicker S, Bhoola K (2004) Colon cancer: genomics and apoptotic events. Biol Chem 385: 449-64

2. Ribic CM, Sargent DJ, Moore MJ, Thibodeau SN, French AJ, Goldberg RM, Hamilton SR, Laurent-Puig P, Gryfe R, Shepherd LE, Tu D, Redston M, Gallinger S (2003) Tumor microsatellite-instability status as a predictor of benefit from fluorouracil-based adjuvant chemotherapy for colon cancer. N Engl J Med 349: 24757

3. Bhushan S, McLeod H, Walko CM (2009) Role of pharmacogenetics as predictive biomarkers of response and/or toxicity in the treatment of colorectal cancer. Clin Colorectal Cancer 8: 15-21

4. Mader RM, Muller M, Steger GG (1998) Resistance to 5-fluorouracil. Gen Pharmacol 31: 661-6

5. Huerta S, Goulet EJ, Livingston EH (2006) Colon cancer and apoptosis. Am J Surg 191: $517-26$

6. Davies JM, Goldberg RM (2008) First-line therapeutic strategies in metastatic colorectal cancer. Oncology (Williston Park) 22: 1470-9

7. Jover R, Zapater P, Castells A, Llor X, Andreu M, Cubiella J, Pinol V, Xicola RM, Bujanda L, Rene JM, Clofent J, Bessa X, Morillas JD, Nicolas-Perez D, Paya A, Alenda C (2006) Mismatch repair status in the prediction of benefit from adjuvant fluorouracil chemotherapy in colorectal cancer. Gut 55: 848-55

8. Warusavitarne J, Schnitzler M (2007) The role of chemotherapy in microsatellite unstable (MSI-H) colorectal cancer. Int J Colorectal Dis 22: 739-48

9. Soreide K, Janssen EA, Soiland H, Korner H, Baak JP (2006) Microsatellite instability in colorectal cancer. Br J Surg 93: 395-406

10. Sinicrope FA, Sargent DJ (2009) Clinical implications of microsatellite instability in sporadic colon cancers. Curr Opin Oncol 21: 369-73

11. Seruca R, Velho S, Oliveira C, Leite M, Matos P, Jordan P (2009) Unmasking the role of KRAS and BRAF pathways in MSI colorectal tumors. Expert Rev Gastroenterol Hepatol 3: 5-9

12. Benhattar J, Cerottini JP, Saraga E, Metthez G, Givel JC (1996) p53 mutations as a possible predictor of response to chemotherapy in metastatic colorectal carcinomas. Int J Cancer 69: 190-2

13. Violette S, Poulain L, Dussaulx E, Pepin D, Faussat AM, Chambaz J, Lacorte JM, Staedel C, Lesuffleur T (2002) Resistance of colon cancer cells to long-term 5fluorouracil exposure is correlated to the relative level of $\mathrm{Bcl}-2$ and $\mathrm{Bcl}-\mathrm{X}(\mathrm{L})$ in addition to Bax and p53 status. Int J Cancer 98: 498-504

14. Bunz F, Hwang PM, Torrance C, Waldman T, Zhang Y, Dillehay L, Williams J, Lengauer C, Kinzler KW, Vogelstein B (1999) Disruption of p53 in human cancer cells alters the responses to therapeutic agents. J Clin Invest 104: 263-9

15. Adamsen BL, Kravik KL, Clausen OP, De Angelis PM (2007) Apoptosis, cell cycle progression and gene expression in TP53-depleted HCT116 colon cancer cells in response to short-term 5-fluorouracil treatment. Int J Oncol 31: 1491-500

16. Mori S, Ogata Y, Shirouzu K (2004) Biological features of sporadic colorectal carcinoma with high-frequency microsatellite instability: special reference to tumor proliferation and apoptosis. Int J Clin Oncol 9: 322-9

17. Hector S, Prehn JH (2009) Apoptosis signaling proteins as prognostic biomarkers in colorectal cancer: A review. Biochim Biophys Acta 1795: 117-29 
18. Aggarwal BB, Shishodia S (2006) Molecular targets of dietary agents for prevention and therapy of cancer. Biochem Pharmacol 71: 1397-421

19. Ramos S (2008) Cancer chemoprevention and chemotherapy: dietary polyphenols and signalling pathways. Mol Nutr Food Res 52: 507-26

20. Subapriya Rajamanickam RA (2008) Natural products and colon cancer: current status and future prospects. Drug Dev Res 69: 460-471

21. Wagner EF, Nebreda AR (2009) Signal integration by JNK and p38 MAPK pathways in cancer development. Nat Rev Cancer 9: 537-49

22. Liu J, Lin A (2005) Role of JNK activation in apoptosis: a double-edged sword. Cell Res 15: 36-42

23 Coulthard LR, White DE, Jones DL, McDermott MF, Burchill SA (2009) p38(MAPK): stress responses from molecular mechanisms to therapeutics. Trends Mol Med 15: 369-79

24. Xavier CP, Lima CF, Preto A, Seruca R, Fernandes-Ferreira M, Pereira-Wilson C (2009) Luteolin, quercetin and ursolic acid are potent inhibitors of proliferation and inducers of apoptosis in both KRAS and BRAF mutated human colorectal cancer cells. Cancer Lett 281: 162-70

25. Gayet J, Zhou XP, Duval A, Rolland S, Hoang JM, Cottu P, Hamelin R (2001) Extensive characterization of genetic alterations in a series of human colorectal cancer cell lines. Oncogene 20: 5025-32

26. Barbour KW, Berger FG (2008) Cell death in response to antimetabolites directed at thymidylate synthase. Cancer Chemother Pharmacol 61: 189-201

27. Bunz F, Dutriaux A, Lengauer C, Waldman T, Zhou S, Brown JP, Sedivy JM, Kinzler KW, Vogelstein B (1998) Requirement for p53 and p21 to sustain G2 arrest after DNA damage. Science 282: 1497-501

28. Preto A, Figueiredo J, Velho S, Ribeiro A, Soares P, Oliveira C, Seruca R (2008) BRAF provides proliferation and survival signals in MSI colorectal carcinoma cells displaying BRAF(V600E) but not KRAS mutations. J Pathol 214: 320-7

29. Itoh N, Semba S, Ito M, Takeda H, Kawata S, Yamakawa M (2002) Phosphorylation of $\mathrm{Akt} / \mathrm{PKB}$ is required for suppression of cancer cell apoptosis and tumor progression in human colorectal carcinoma. Cancer 94: 3127-34

30. Osaki M, Oshimura M, Ito H (2004) PI3K-Akt pathway: its functions and alterations in human cancer. Apoptosis 9: 667-76

31. Rychahou PG, Jackson LN, Silva SR, Rajaraman S, Evers BM (2006) Targeted molecular therapy of the PI3K pathway: therapeutic significance of PI3K subunit targeting in colorectal carcinoma. Ann Surg 243: 833-42; discussion 843-4

32. Thompson N, Lyons $\mathbf{J}$ (2005) Recent progress in targeting the Raf/MEK/ERK pathway with inhibitors in cancer drug discovery. Curr Opin Pharmacol 5: 350-6

33. McCubrey JA, Steelman LS, Abrams SL, Lee JT, Chang F, Bertrand FE, Navolanic PM, Terrian DM, Franklin RA, D'Assoro AB, Salisbury JL, Mazzarino MC, Stivala F, Libra M (2006) Roles of the RAF/MEK/ERK and PI3K/PTEN/AKT pathways in malignant transformation and drug resistance. Adv Enzyme Regul 46: 249-79

34. Tang XY, Zhu YQ, Tao WH, Wei B, Lin XL (2007) Synergistic effect of triptolide combined with 5-fluorouracil on colon carcinoma. Postgrad Med J 83: 338-43

35. Zhang YQ, Tang XQ, Sun L, Dong L, Qin Y, Liu HQ, Xia H, Cao JG (2007) Rosiglitazone enhances fluorouracil-induced apoptosis of HT-29 cells by activating peroxisome proliferator-activated receptor gamma. World J Gastroenterol 13: 1534-40

36. Wang CZ, Luo X, Zhang B, Song WX, Ni M, Mehendale S, Xie JT, Aung HH, He TC, Yuan CS (2007) Notoginseng enhances anti-cancer effect of 5-fluorouracil on human colorectal cancer cells. Cancer Chemother Pharmacol 60: 69-79 
37. Shi R, Huang Q, Zhu X, Ong YB, Zhao B, Lu J, Ong CN, Shen HM (2007) Luteolin sensitizes the anticancer effect of cisplatin via c-Jun NH2-terminal kinase-mediated p53 phosphorylation and stabilization. Mol Cancer Ther 6: 1338-47

38. Hou Q (2008) [Effect of staurosporine induced apoptosis of MCF7/GFP-Bax stable cell line on Bax translocation from cytosol into mitochondria]. Yao Xue Xue Bao 43: $378-82$ 


\section{Figure legends}

Figure 1 - Effect on cell viability/proliferation (a) and apoptosis (b) of different concentrations of 5-fluorouracil (5-FU), for 48h, in HCT15 and CO115 colon cancer cells, using MTT and TUNEL assay, respectively. The MTT value in the beginning of the assay $(\mathrm{t}=$ 0h) was subtracted from all experimental conditions at $48 \mathrm{~h}$. Negative values in MTT assay indicate induction of severe and acute cell toxicity after $48 \mathrm{~h}$ incubation with test compound. Results are presented as mean \pm SEM of at least 3 independent experiments. ${ }^{*} \mathrm{P} \leq 0.05$, ** $\mathrm{P} \leq$ 0.01 and $* * * \mathrm{P} \leq 0.001$.

Figure 2 - Effect on apoptosis by 5-fluorouracil (FU) 500, 100 and $1 \mu \mathrm{M}$, quercetin $12 \mu \mathrm{M}$ (Q12) and luteolin $12 \mu \mathrm{M}$ (L12) alone, as well as the natural compounds co-incubated with FU for 48h, in HCT15 (a) and CO115 (b) cells, using TUNEL assay. Results are presented as mean \pm SEM of at least 3 independent experiments. $* * * \mathrm{P} \leq 0.001$, when compared with control; $++\mathrm{P} \leq 0.01$, when compared with the respective natural compound alone; \#\# $\mathrm{P} \leq 0.01$ and \#\#\# $\mathrm{P} \leq 0.001$, when compared with FU alone; NS, not significant differences observed between each other.

Figure 3 - Effect of a caspase inhibitor zVAD-FMK (zVAD) $20 \mu \mathrm{M}$ on the apoptosis induction by quercetin $12 \mu \mathrm{M}(\mathrm{Q} 12)$, luteolin $12 \mu \mathrm{M}$ (L12) and staurosporine (STS) $0.25 \mu \mathrm{M}$, for 48h, in HCT15 (a) and CO115 (b) cells, using TUNEL assay. Results are presented as mean \pm SEM of at least 3 independent experiments. * $\mathrm{P} \leq 0.05$, *** $\mathrm{P} \leq 0.001$, when compared to control; $++\mathrm{P} \leq 0.01$ and $+++\mathrm{P} \leq 0.001$, when compared with the respective compound alone; \#\# $\mathrm{P} \leq 0.01$ and \#\#\# $\mathrm{P} \leq 0.001$, when compared with $\mathrm{zVAD}$ alone; NS, not significant differences observed between each other. Effects on caspase-9, caspase-3 and PARP-1 expressions, for 48h, of Q, L, 5-FU (FU) and STS alone, in HCT15 and CO115 cells (c), by western blot. Images are representative of at least 3 independent experiments.

Figure 4 - Effects on p53, Bax and Bcl-2 (a), phospho-JNK and total JNK (b) and phosphop38 and total P38 (c) expressions, for 48h, of quercetin $12 \mu \mathrm{M}$ (Q12), luteolin $12 \mu \mathrm{M}$ (L12), staurosporine (STS) $0.25 \mu \mathrm{M}$ and 5-fluorouracil (FU) $1 \mu \mathrm{M}$ and $100 \mu \mathrm{M}$, in HCT15 and CO115 cells, by western blot. Images are representative of at least 3 independent experiments. 
Figure 5 - Effects on caspase-9, caspase-3, PARP-1, p53 and Bcl-2 expressions, for 48h, of co-incubation of quercetin $12 \mu \mathrm{M}(\mathrm{Q} 12)$ and 5-fluorouracil $1 \mu \mathrm{M}(\mathrm{FU} 1)$ in CO115 cells, by western blot. Images are representative of at least 3 independent experiments.

Figure 6 - Dependence on p53 for apoptosis induction by quercetin (Q) and 5-fluorouracil (FU). (a) CO115 cells knockdown for p53 by siRNA (100nM) were incubated with FU $1 \mu \mathrm{M}$ (FU1) or Q $12 \mu \mathrm{M}(\mathrm{Q} 12)$ alone, and with both in combination for $48 \mathrm{~h}$ and apoptosis assessed by TUNEL assay. Control cells were transfected with control siRNA. Compounds were added 24h after transfection. p53 knockdown efficiency was monitored by western blot (inset). (b) HCT116 p53-wt (p53 +/+) and HCT116 p53-null (p53 -/-) cells were used to observe effects of FU, Q and both in combination on apoptosis after $48 \mathrm{~h}$ of incubation, as assessed by TUNEL assay. FU10: FU 10 $\mu$ M; FU100: FU 100 $\mu$ M; Q15: Q 15 $\mu$. (a; b) Results are presented as mean $\pm \mathrm{SEM}$ of at least 3 independent experiments. $* \mathrm{P} \leq 0.05$, ** $\mathrm{P} \leq 0.01$ and $* * * \mathrm{P} \leq 0.001$, when compared with control; + $\mathrm{P} \leq 0.05$, when compared with each other; \#\#\# $\mathrm{P} \leq 0.001$, when compared with respective FU alone; $\varnothing \mathrm{P} \leq 0.05$, when compared with respective Q alone; NS, not significant differences observed between each other. 


\section{A MTT reduction test}

HCT15

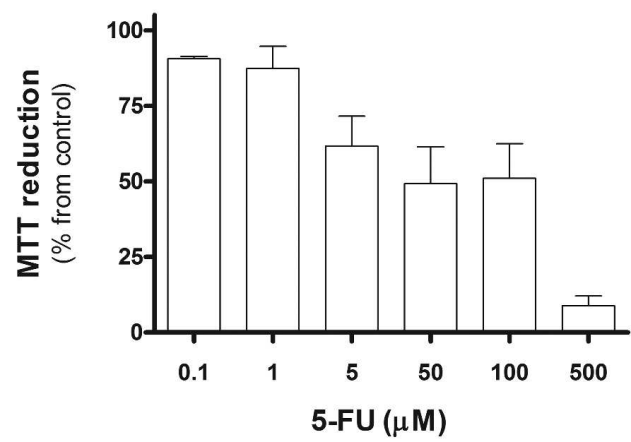

\section{B TUNEL assay}

HCT15

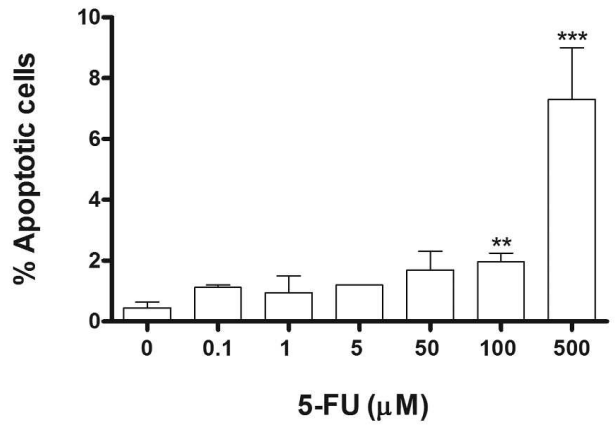

C0115

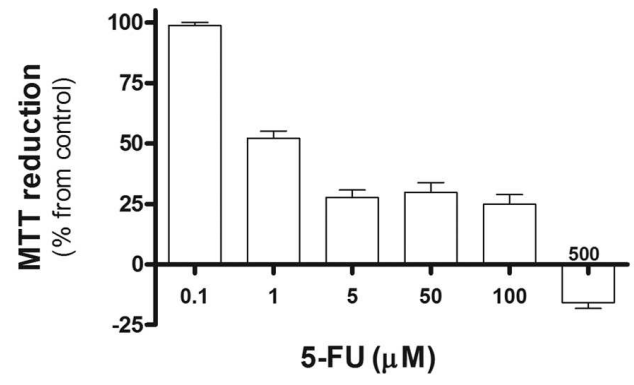

C0115

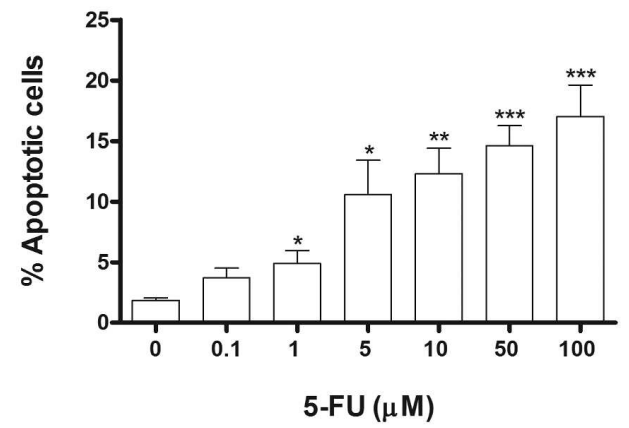

Figure 1 - Effect on cell viability/proliferation (a) and apoptosis (b) of different concentrations of 5fluorouracil (5-FU), for 48h, in HCT15 and CO115 colon cancer cells, using MTT and TUNEL assay, respectively. The MTT value in the beginning of the assay $(t=0 h)$ was subtracted from all experimental conditions at $48 \mathrm{~h}$. Negative values in MTT assay indicate induction of severe and acute cell toxicity after $48 \mathrm{~h}$ incubation with test compound. Results are presented as mean \pm SEM of at least 3 independent experiments. * $\mathrm{P} \leq 0.05, * * \mathrm{P} \leq 0.01$ and $* * * \mathrm{P} \leq 0.001$. 


\section{TUNEL assay}

A HCT15

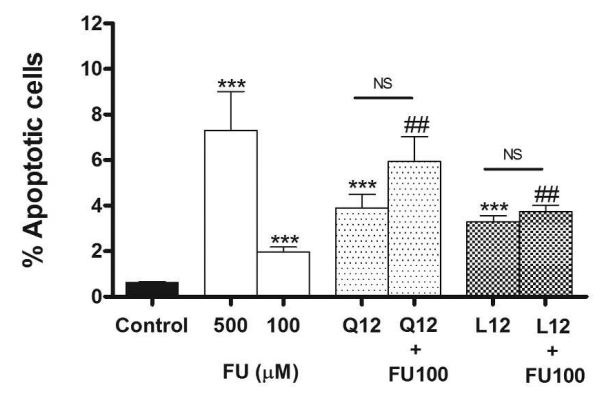

B C0115

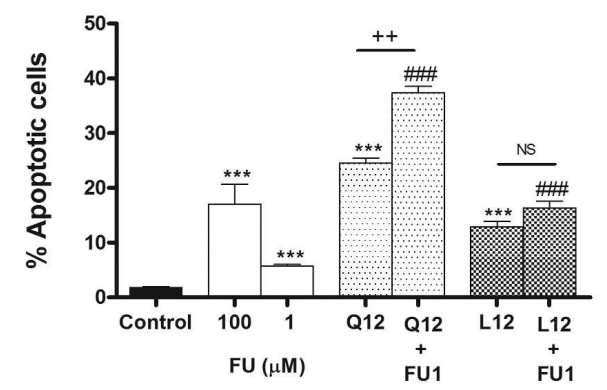

Figure 2 - Effect on apoptosis by 5-fluorouracil (FU) 500, 100 and $1 \mu \mathrm{M}$, quercetin $12 \mu \mathrm{M}(\mathrm{Q} 12)$ and luteolin $12 \mu \mathrm{M}$ (L12) alone, as well as the natural compounds co-incubated with FU for 48h, in HCT15

(a) and CO115 (b) cells, using TUNEL assay. Results are presented as mean \pm SEM of at least 3 independent experiments. $* * * \mathrm{P} \leq 0.001$, when compared with control; $++\mathrm{P} \leq 0.01$, when compared with the respective natural compound alone; \# $\mathrm{P} \leq 0.01$ and \#\# \# $\mathrm{P} \leq 0.001$, when compared with FU alone; NS, not significant differences observed between each other. $160 \times 74 \mathrm{~mm}(600 \times 600 \mathrm{DPI})$ 
A HCT15

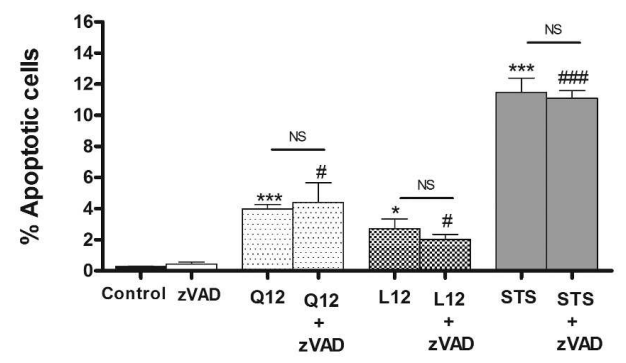

B C0115

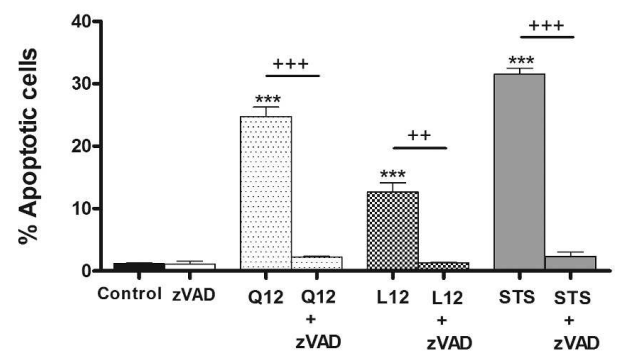

c0115

$\mathrm{C}$

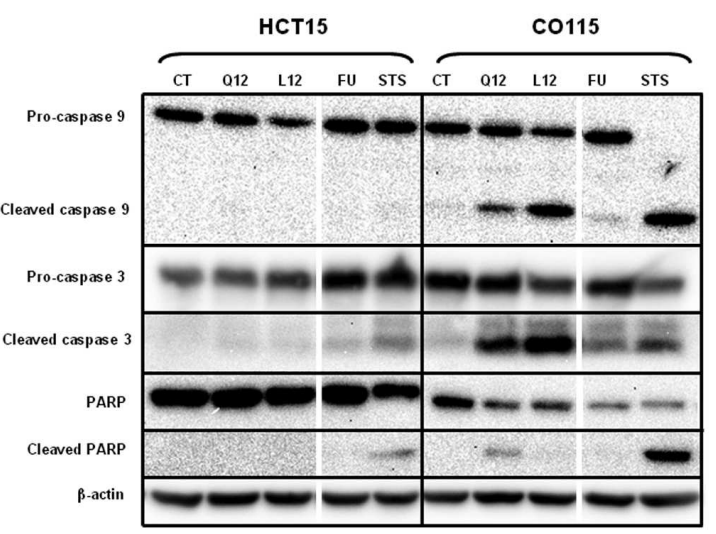

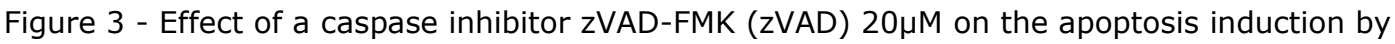
quercetin $12 \mu \mathrm{M}(\mathrm{Q} 12)$, luteolin $12 \mu \mathrm{M}$ (L12) and staurosporine (STS) $0.25 \mu \mathrm{M}$, for $48 \mathrm{~h}$, in HCT15 (a) and CO115 (b) cells, using TUNEL assay. Results are presented as mean \pm SEM of at least 3 independent experiments. ${ }^{*} \mathrm{P} \leq 0.05, * * * \mathrm{P} \leq 0.001$, when compared to control; $++\mathrm{P} \leq 0.01$ and $+++\mathrm{P} \leq 0.001$, when compared with the respective compound alone; \#\# $\mathrm{P} \leq 0.01$ and \#\#\# $\mathrm{P} \leq$ 0.001 , when compared with ZVAD alone; NS, not significant differences observed between each other. Effects on caspase-9, caspase-3 and PARP-1 expressions, for 48h, of Q, L, 5-FU (FU) and STS alone, in HCT15 and CO115 cells (c), by western blot. Images are representative of at least 3 independent experiments. $155 \times 128 \mathrm{~mm}(600 \times 600 \mathrm{DPI})$ 


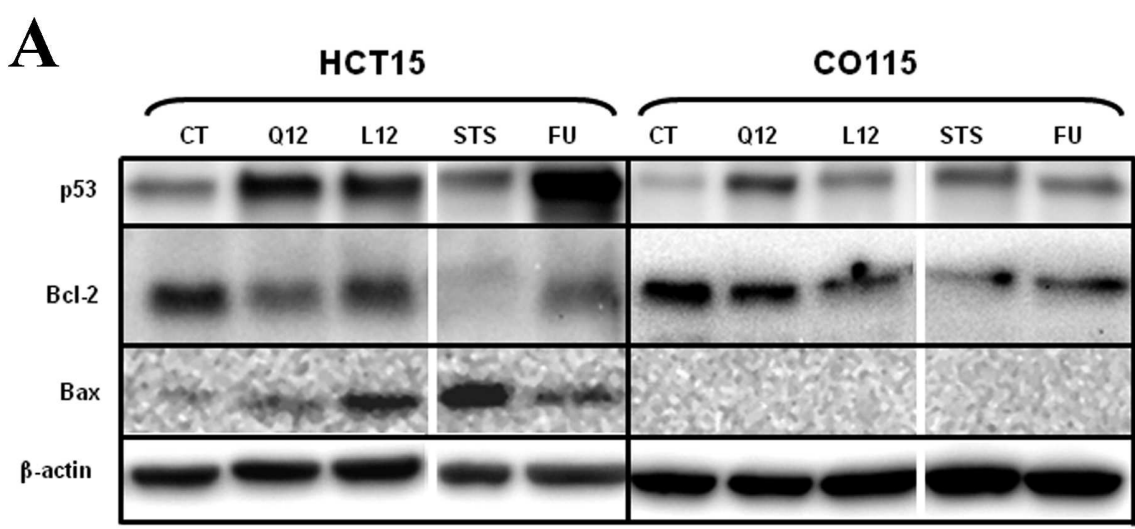

B
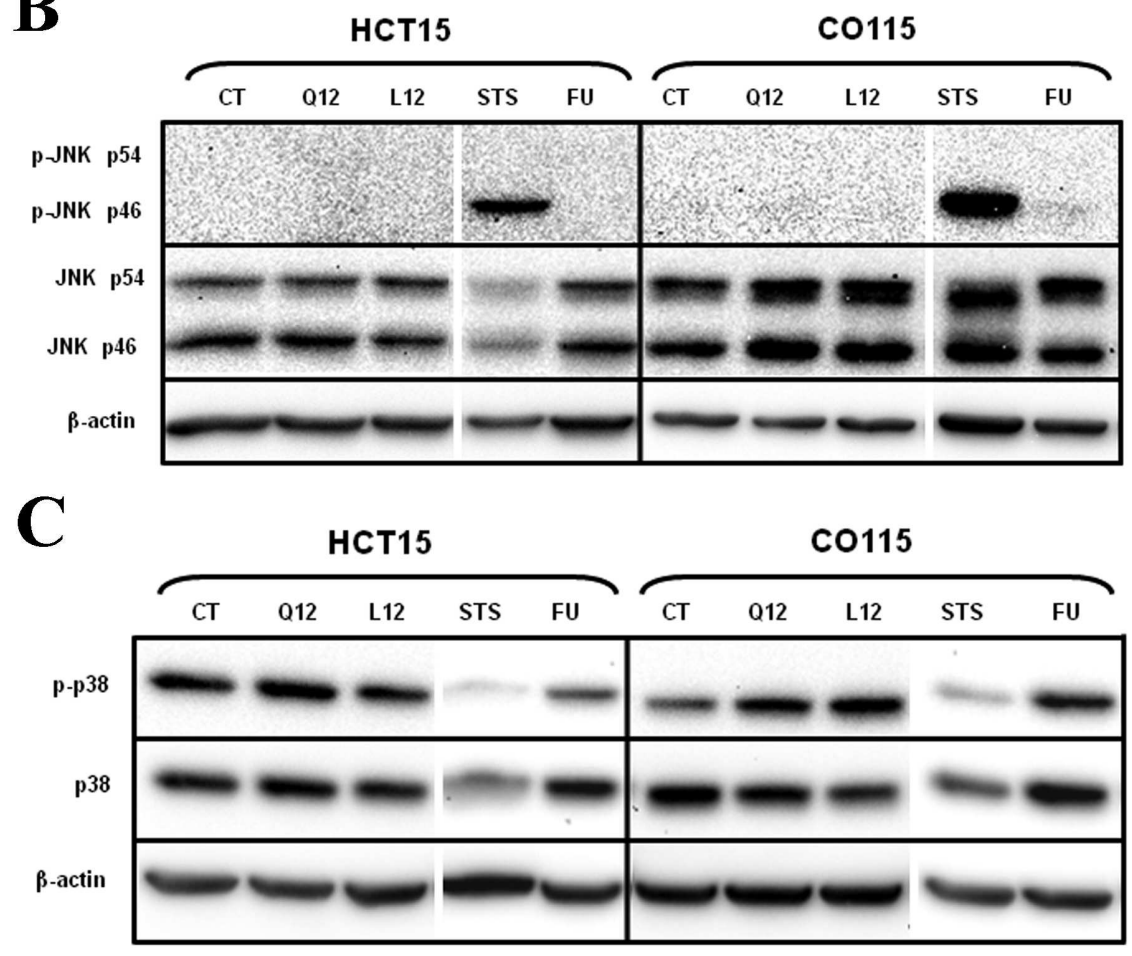

Figure 4 - Effects on p53, Bax and Bcl-2 (a), phospho-JNK and total JNK (b) and phospho-p38 and

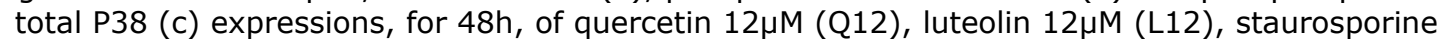
(STS) $0.25 \mu \mathrm{M}$ and 5-fluorouracil (FU) $1 \mu \mathrm{M}$ and $100 \mu \mathrm{M}$, in HCT15 and CO115 cells, by western blot. Images are representative of at least 3 independent experiments.

$80 \times 106 \mathrm{~mm}(600 \times 600 \mathrm{DPI})$ 


\section{C0115}

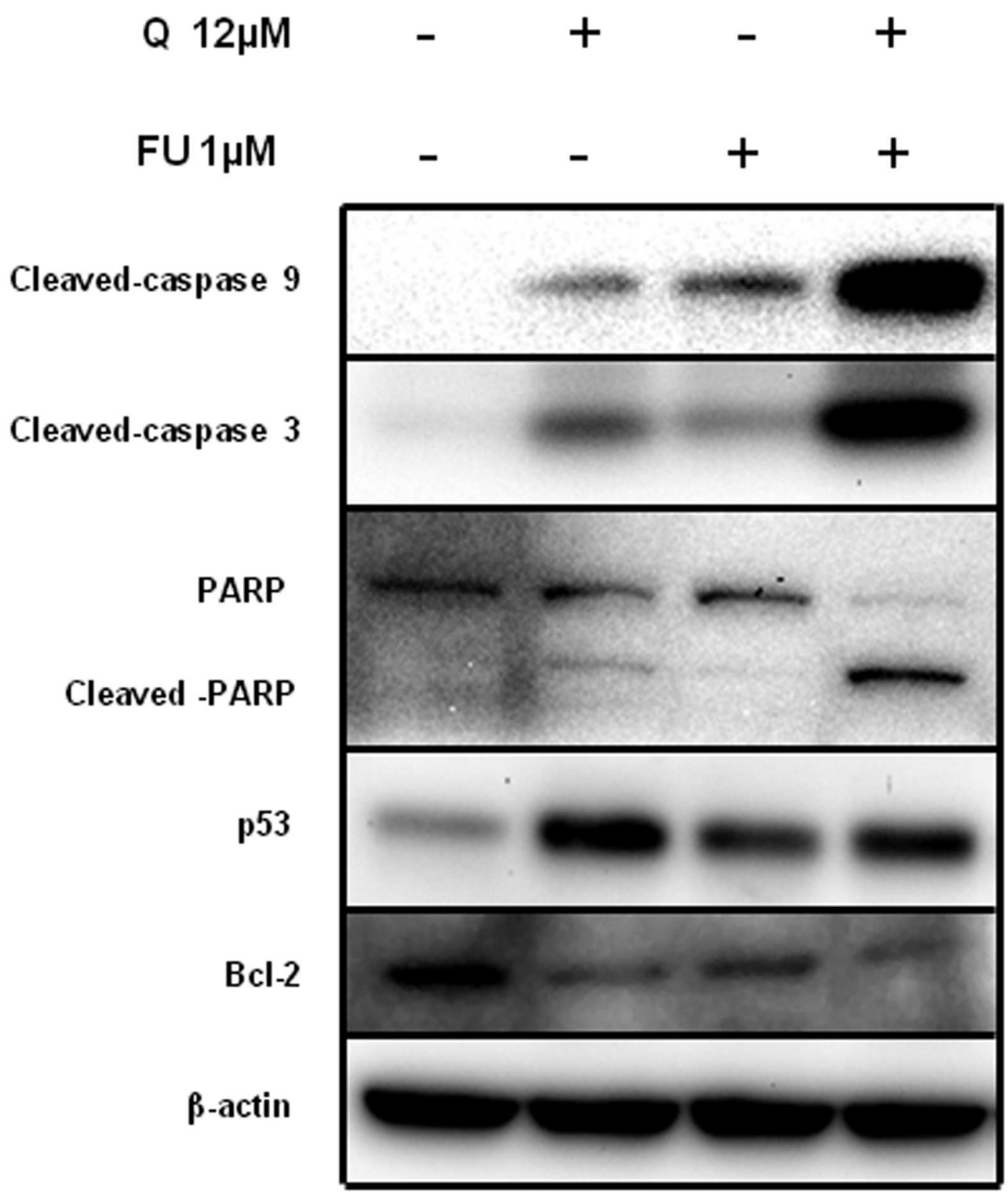

Figure 5 - Effects on caspase-9, caspase-3, PARP-1, p53 and Bcl-2 expressions, for $48 \mathrm{~h}$, of coincubation of quercetin $12 \mu \mathrm{M}(\mathrm{Q} 12)$ and 5-fluorouracil $1 \mu \mathrm{M}$ (FU1) in CO115 cells, by western blot. Images are representative of at least 3 independent experiments.

$157 \times 191 \mathrm{~mm}(600 \times 600 \mathrm{DPI})$ 
A

C0115
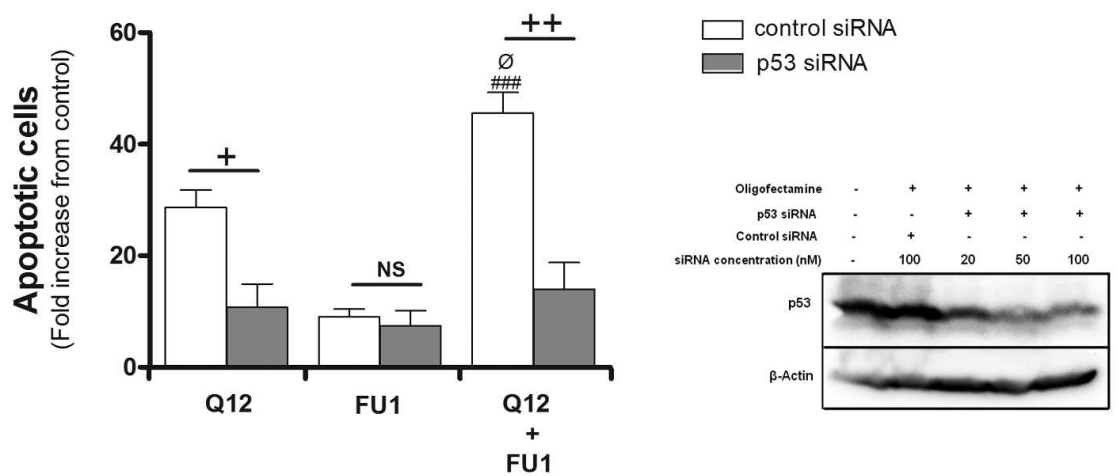

B

HCT116 p53 +/+

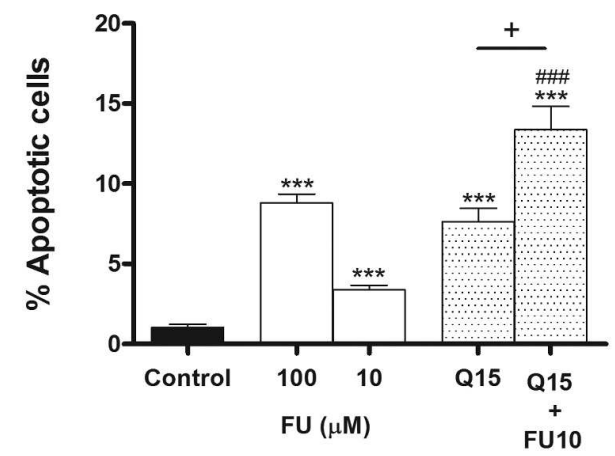

HCT116 p53 -/-

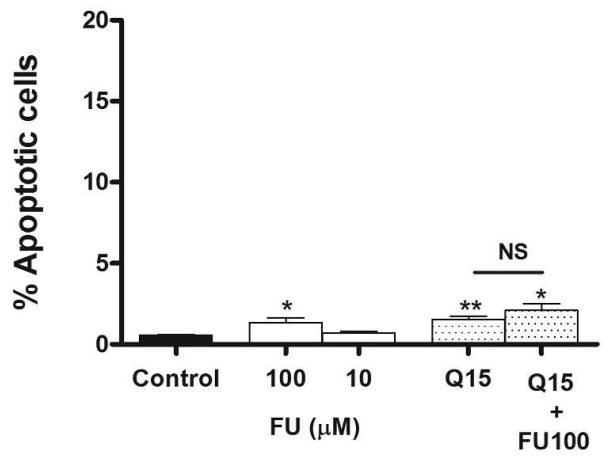

Figure 6 - Dependence on p53 for apoptosis induction by quercetin (Q) and 5-fluorouracil (FU). (a) CO115 cells knockdown for p53 by siRNA (100nM) were incubated with FU $1 \mu M$ (FU1) or Q $12 \mu M$ (Q12) alone, and with both in combination for 48h using, and apoptosis assessed by TUNEL assay. Control cells were transfected with control siRNA. Compounds were added $24 \mathrm{~h}$ after transfection. p53 knockdown efficiency was monitored by western blot (inset). (b) HCT116 p53-wt (p53 +/+) and HCT116 p53-null (p53 -/-) cells were used to observe effects of FU, Q and both in combination on apoptosis after $48 \mathrm{~h}$ of incubation, as assessed by TUNEL assay. FU10: FU 10 $\mu$ M; FU100: FU

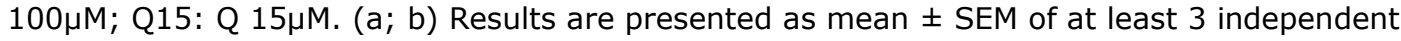
experiments. * $\mathrm{P} \leq 0.05, * * \mathrm{P} \leq 0.01$ and $* * * \mathrm{P} \leq 0.001$, when compared with control; $+\mathrm{P} \leq 0.05$, when compared with each other; \#\#\# P $\leq 0.001$, when compared with respective FU alone; $\varnothing \mathrm{P} \leq$ 0.05 , when compared with respective Q alone; NS, not significant differences observed between each other.

$139 \times 147 \mathrm{~mm}(600 \times 600 \mathrm{DPI})$ 